\title{
Characterization and Mitigation of Multipath for Terrestrial based Aviation Radionavigation
}

\author{
Nicolas Schneckenburger, Sherman Lo, Robert Lilley, Uwe-Carsten Fiebig, \\ Per Enge
}

\begin{abstract}
In this contribution a comprehensive overview of multipath propagation and its effects on current and future L-band terrestrial radionavigation devices is given. The line of sight and multipath propagation between a ground station transmitter and an aircraft is characterized based on measurement data. The paper presents different methods to mitigate effects of multipath propagation and limit the resulting errors.
\end{abstract}

\section{INTRODUCTION}

The communication, navigation, and surveillance (CNS) infrastructure used to support the airspace is currently undergoing a major innovation process. Legacy systems, some dating back to the 1950s, are being replaced or superseded by new, more efficient systems. The change is necessary as the future airspace will need to support higher traffic levels and require more efficient flight operations. The navigation capabilities needed to support these increased demands will primarily come from global navigation satellite system (GNSS). Nevertheless, ground-based radionavigation systems will still play a vital role as alternative positioning, navigation, and timing (APNT) system in the future navigation infrastructure [1]. APNT systems are used as backup systems in case the primary means of navigation using GNSS becomes unavailable as a result of intentional or unintentional interference or system failures.

Indeed terrestrial radionavigation will continue to be a component of CNS. Current US and European plans are to continue and even expand the operations of distance measuring equipment (DME), a two-way ranging system originating in the 1950s [2]. Additionally, more modern aviation radio signals such as L-band Digital Aeronautical Communication System (LDACS) or automatic dependent surveillance - broadcast (ADS-B) [3], [4] may be used to enhance terrestrial radionavigation. These systems offer both more efficient bandwidth utilization along with passive ranging. These features can improve capacity, ranging, coverage, and security [5].

Improvements to terrestrial radionavigation capabilities are vital if the systems are to support many of the flight operations enabled by GNSS. New flight procedures and a more crowded airspace require performance-based navigation (PBN) and demand an improved ranging performance as well as integrity. For example, DME is specified by International Civil Aviation Organization (ICAO) to be a $1.0 \mathrm{nmi}$ (nautical mile) positioning system [6]. However, to be relevant for the coming airspace, it needs to be capable of supporting area navigation (RNAV) and required navigation performance (RNP) into terminal airspace which means $0.3 \mathrm{nmi}$ and better performance with commensurate integrity. And so, a major challenge is to make sure that APNT systems can support the performance requirements on accuracy, integrity, capacity and coverage to support safe and efficient airspace operations in case of a GNSS outage [7].

The main limiting factors in terms of accuracy and integrity for ground-based radionavigation systems are the propagation characteristics between the aircraft and ground station. Throughout this paper we refer to those propagation characteristics as the air-ground (AG) channel. Of the AG 


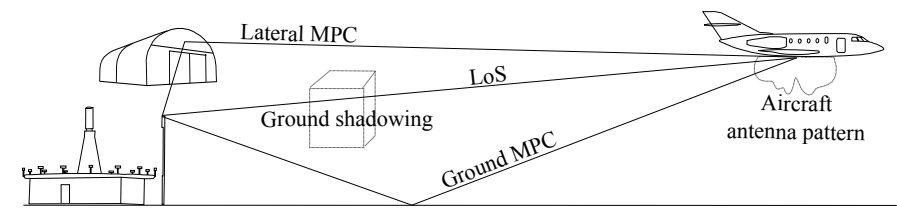

Fig. 1. Multipath propagation in the context of aircraft navigation.

channel the major factor causing large ranging errors, and thus prohibiting accuracy and integrity needed for RNP and RNAV 0.3 operations is multipath propagation. Although only very few scenarios have been investigated in detail, large ranging errors exceeding $200 \mathrm{~m}$ have been observed [7], [8]. Unfortunately it is too expensive and complex to extensively investigate further scenarios covering the large diversity of regions and situations aircraft require precise navigation.

Investigating the AG channel and in particular multipath propagation is essential for the analysis and mitigation of ranging errors. The current state of the art solution to investigate the propagation characteristics of a radio channel is wide-band measurement. Only very few of such measurements have been conducted in the past [9], [10] in the context of the navigation application. The vast majority of measurements have been conducted focusing on communication applications [11]-[14]. A comprehensive characterization of the propagation characteristics relevant for ground-based radionavigation based on measurement data is given in this paper.

The main novelty brought forward in this paper is two-fold. First, the paper systematically characterizes the channel and identifies main factors leading to significant multipath propagation induced errors. A combination of measurement data collected over several campaigns previously mentioned, processing, analysis, and previous work is synthesized to perform the characterization. Second, the paper identifies and illustrates mitigations feasible and suitable for terrestrial radionavigation signals used for aviation.

The paper is organized as follows: Sec. II provides an overview of the different propagation effects in the AG radio channel and characterize their effect on ranging. The different multipath and nonmultipath propagation effects are described in Sec. II. The influence of the AG channel on ranging is discussed in Sec. III. Sec. IV gives an overview of the different mitigation techniques. Sec. V describes the effectiveness of the mitigation techniques given different propagation conditions. In Sec. VI conclusions are drawn and a way forward to improve the performance of ground-based navigation systems is outlined.

\section{The AIR-GRound CHANNEL}

This section provides an overview of the fundamental propagation effects of the AG channel. Fig. 1 shows the main propagation paths of this channel: on its way from the transmitter to the receiver the transmitted signal may travel (1) on the so-called line-of-sight (LoS) path which exists if ground station and aircraft see each other; (2) on a path which contains a reflection point on the ground; (3) on paths which contain reflection points on buildings, trees, and other objects.

Associated with these paths are the following signal components: (1) the LoS signal, (2) the ground multipath component (MPC), and (3) the lateral MPC.

At the receiver all signal components are superimposed. The AG channel typically exhibits multipath propagation, i.e., the receive signal consists of several components which even may not encompass the LoS signal.

Eventually, the antenna patterns both at the ground station and at the aircraft have a strong influence, e.g., on the receive power of the various signal components. In Fig. 1 only the antenna 
pattern at the aircraft is taken into account for as the antenna pattern at the ground site typically does not exhibit strong variations in the azimuthal plane.

Note that transmitter and receiver might be exchanged with no influence on the above mentioned propagation paths.

The different signal components can be characterized using different parameters. The most important parameters for ranging are:

- Propagation delay is the time it takes for a signal to travel from transmitter to the receiver.

- Receive power is understood as the power of the respective signal component received at the antenna.

- Doppler frequency is a frequency shift of the signal's carrier frequency with respect to the transmit carrier frequency due to the changing link distance. The Doppler frequency is proportional to the rate of change of the propagation delay.

- Lifetime of a signal component is the time span during which the signal component is detectable at the receiver.

\section{A. Line-of-sight Signal}

If transmitter and receiver see each other, there exists a LoS path along which the electromagnetic signal can directly travel. The LoS signal is characterized as follows:

- Propagation delay $\tau_{\text {LoS }}$ : The LoS signal exhibits a propagation delay - referred to as LoS propagation delay - which is determined by the speed of light and by the link distance. For AG propagation the speed of light depends on the composition of the troposphere and is slightly less than the speed of light in the vacuum.

- Receive power $P_{\mathrm{LoS}}$ : The receive power of the LoS signal - also denoted as LoS receive power - follows the law of free space propagation if the Fresnel zone is free of obstacles, i.e., the LoS receive power is proportional to the inverse of the square of the link distance. At large distances between the aircraft and the ground station the curvature of the Earth causes the LoS path to vanish. Nevertheless, the aircraft may still receive a signal since it travels as ground wave along the surface of the Earth. Beyond LoS propagation, however, is not treated in the following, since radionavigation techniques of interest in this paper are not designed to operate in such a situation.

- Doppler frequency $\nu_{\mathrm{LoS}}$ : The Doppler frequency of the LoS signal - in short: LoS Doppler frequency - is determined by the velocity of the aircraft relative to the location of the airport.

The LoS signal is the most desired signal for radionavigation. Ideally, only the LoS signal exists, i.e., the receiver only receives the LoS signal and, hence, does not have to cope with degradations due to MPCs. Thus, the LoS signal is used as a reference signal.

With respect to this reference signal three further parameters are introduced which relate propagation delay $\tau_{\mathrm{M}}$, receive power $P_{\mathrm{M}}$, and Doppler frequency $\nu_{\mathrm{M}}$ of an MPC to LoS propagation delay, LoS receive power and LoS Doppler frequency, respectively. Accordingly, these parameters are denoted $\tau_{\mathrm{M}, \mathrm{rel}}, P_{\mathrm{M}, \mathrm{rel}}, \nu_{\mathrm{M}, \mathrm{rel}}$ and are defined by

- relative propagation delay $\tau_{\mathrm{M} \text {,rel }}=\tau_{\mathrm{M}}-\tau_{\mathrm{LoS}}$,

- relative receive power $P_{\mathrm{M}, \mathrm{rel}}=P_{\mathrm{M}} / P_{\mathrm{LoS}}$, and

- relative Doppler frequency $\nu_{\mathrm{M}, \mathrm{rel}}=\nu_{\mathrm{M}}-\nu_{\mathrm{LoS}}$.

\section{B. Ground Multipath Component}

A signal component which travels from transmitter to receiver via a reflection off the ground is denoted as ground MPC. It is characterized as follows: 
- Propagation delay $\tau_{\mathrm{M}}$ : A ground MPC is typically reflected off the ground at a very shallow angle. The low angle is due to the typically small ratio of the aircraft's altitude to the aircraft's distance from the ground station. This geometrical situation causes the ground MPC delay to be only slightly larger than the LoS path delay in most flight situations. This small difference in path length translates to a likewise small difference in terms of propagation delay, i.e., $\tau_{\mathrm{M}}$ is only slightly larger than $\tau_{\mathrm{LoS}}$, resulting in a very small relative propagation delay $\tau_{\mathrm{M} \text {,rel }}$ of the ground MPC.

- Receive power $P_{\mathrm{M}}$ : Due to the shallow angle at which the ground MPC is reflected, the reflection coefficient is often approaching 1 , causing $P_{\mathrm{M}}$ to be in a similar order than $P_{\mathrm{LoS}}$. Hence, the relative receive power $P_{\mathrm{M} \text {,rel }}$ can often approach $0 \mathrm{~dB}$.

- Doppler frequency $\nu_{\mathrm{M}}$ : Following above described geometrical situation it is obvious that $\nu_{\mathrm{M}}$ is very close to $\nu_{\mathrm{LoS}}$, i.e., the relative Doppler frequency $\nu_{\mathrm{M} \text {,rel }}$ of the ground MPC is close to zero.

- Lifetime: A ground MPC can exist over a long time for two connected reasons: the size of the ground area is typically quite large, and the velocity of the reflection point over this ground area is relatively small [15]; note that the movement of the reflection point is determined by the geometrical situation of the moving aircraft and the ground station location. Thus, a small patch of a well reflecting surface can cause an aircraft to receive a ground MPC over a long period.

\section{Lateral Multipath Components}

A lateral MPC arises, when a part of the emitted signal is reflected via one or more reflection points which lay on the surface of buildings, trees, terrain, fences and other objects. The lateral MPCs are characterized as follows:

- Propagation delay $\tau_{\mathrm{M}}$ : The delay of a lateral MPC is defined by the duration it takes for the signal to travel from the transmitter via the reflection point(s) towards the receiver. $\tau_{\mathrm{M}}$ is always larger than the LoS delay. The relative propagation delay $\tau_{\mathrm{M} \text {,rel }}$ can be quite large: values of up to $15 \mu \mathrm{s}$ have been observed. These values are obtained when the distance between the reflection point and the LoS path is large. Indeed, in airport environments there exist a large number of lateral MPCs with large values for $\tau_{\mathrm{M} \text {,rel }}$ due to the particular mix of large buildings and open areas [7], [9].

- Receive power $P_{\mathrm{M}}$ : Apart from the link distance, the receive power $P_{\mathrm{M}}$ of a lateral MPC is mainly determined by the complex reflection coefficient (or coefficients for multi-bounce reflections) of the surface it is reflected off. The complex reflection coefficient depends on the material and roughness of the reflecting surface as well as the angle between the incoming signal and the reflecting surface [16]-[18]. If this angle approaches 0 degree, the reflection coefficient becomes 1, i.e., all signal power is reflected; such a situation occurs rarely. From Fig. 2 it can be observed that the lateral MPCs usually have a very small power. An analysis of the reflector locations shows that the most common type of lateral MPC emerges when the signal is reflected via the ground towards a reflecting structure [19], see case (1) in Fig. 3. Due to two successive reflections such a lateral MPC usually has a small receive power. In contrast, the two cases (2) and (3) in Fig. 3 typically result in lateral MPCs with a higher receive power but are typically very rare and of short duration.

- Doppler frequency $\nu_{\mathrm{M}}$ : The Doppler frequency of a lateral MPC depends on the geometry of the scenario, i.e., the flight trajectory of the aircraft, the aircraft's velocity, and the location of the reflection point. In contrast to the Doppler frequency of the ground $\mathrm{MPC}, \nu_{\mathrm{M}, \text { rel }}$ can 


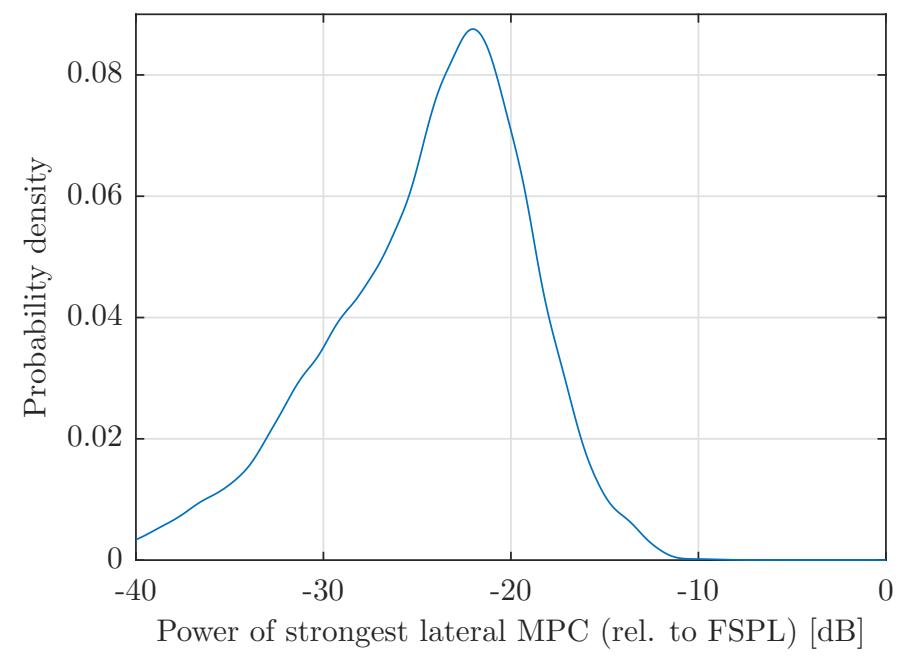

Fig. 2. Distribution of the power of the strongest lateral MPC.

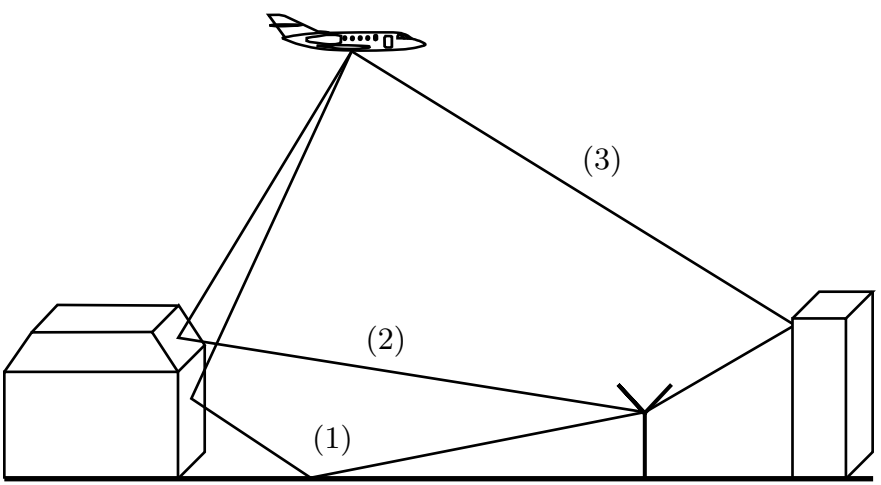

Fig. 3. Creation of three cases of lateral multipath propagation in the AG channel.

vary over a large range. Typical values for the L-band measured for an aircraft flying at 30 $\mathrm{km}$ to the ground stations are in the range between $-10 \mathrm{~Hz}$ and $+10 \mathrm{~Hz}$.

- Lifetime: The majority of the lateral MPCs exists only for a very short time. This is due to the fact that the reflection point usually moves so fast over the reflecting surface that a particular lateral MPC only exists for tens or hundreds of ms. Especially, a lateral MPC originating from a non-vertical surface - case (2) in Fig. 3 - is expected to last only for a very short time due to the usually small area of a non-vertical surface. However, lateral MPCs with durations exceeding several minutes have also been observed in measurements. Such long lasting lateral MPCs usually occur in situations where the aircraft is flying on straight trajectories [10], [19].

Note that often ground stations are situated either directly on the ground or on top of the highest building or tower of an airport. In the first case all types of lateral MPCs are possible, in the latter case lateral MPCs like those of case (3) in Fig. 4 are avoided, since they require a reflection surface above the height of the ground station.

\section{Ground Shadowing}

Ground shadowing is a propagation phenomenon which refers to the blockage of the LoS signal by buildings, vegetation, or the terrain. Ground shadowing usually causes a strong attenuation or 
even an obliteration of the LoS signal. The blocking object has to clearly interrupt the LoS path, i.e. the blocking object is seen from the ground station antenna under the same elevation angle as the aircraft which, however, is behind the blocking object. Ground shadowing by buildings can be avoided by an appropriate choice of the height of the ground station antenna as well as the flight routes. At airports which are located in the vicinity of hills and mountains ground shadowing by terrain may happen quite often.

\section{E. Aircraft Pattern}

The aircraft pattern (or in situ antenna gain pattern) describes the radiation pattern of the aircraft mounted on the airframe [20]. The radiation pattern of a given antenna measured in an anechoic chamber may be very different to the aircraft pattern for a specific aircraft for two reasons. First, mounting the antenna on an irregular surface like an airframe usually alters the shape of the radiation pattern, e.g., additional nulls may be introduced. Second, the airframe may shadow signals depending on the direction the signals are received from. Thus, the airframe shadowing may become especially severe when the aircraft is performing banking maneuvers. Unless the aircraft is close to the ground station (link distance approximately $>10 \mathrm{~km}$ ) the LoS signal and different MPCs arrive at the aircraft from almost the identical direction. Thus, the aircraft pattern influences all signal components in a similar fashion. However, if the aircraft is in the vicinity of the ground station, the different signal components may be received from different directions: thus, the LoS signal may be attenuated while an MPC is not. An overview of the aircraft patterns for different aircraft types is given in [21].

\section{Critical Situations for Radionavigation}

In the following section the most critical propagation conditions in the AG channel producing ranging errors are analyzed.

As these situations often include the presence of an MPC, Sec. A describes how the overlapping of an MPC with the LoS signal results in ranging errors. Sec. B provides examples for situations which cause MPCs with large relative receive power and where, in turn, large ranging errors can be observed.

\section{A. Effect of an $M P C$ on Ranging Errors}

The superposition of the LoS signal with an MPC influences the outcome of the correlation process of the navigation receiver. The MPC causes the maximum of the correlator output to be biased with respect to the value obtained under ideal conditions. The result is a ranging error which almost linearly depends on the bias of the correlator output ${ }^{1}$.

Fig. 4 shows the ranging error envelope for a DME receiver, if the receive signal consists of the LoS signal and one additional MPC with a relative power $P_{\mathrm{M} \text {,rel }}=-6 \mathrm{~dB}$. Depending on the relative phase of the $\mathrm{MPC}$, the ranging error can either be positive or negative. The maximum and minimum of the envelope are obtained if the LoS signal and MPC are perfectly inphase or in opposite phase, respectively. From Fig. 4 it is observed that ranging errors - even for a MPC which is $6 \mathrm{~dB}$ less powerful than the LoS signal - can be large exceeding $100 \mathrm{~m}$ : a significant dependence of the ranging error on the MPC's relative delay $\tau_{\mathrm{M} \text {,rel }}$ can be identified. For small delays, e.g., $\tau_{\mathrm{M} \text {,rel }}<100 \mathrm{~ns}$ which are typical for ground MPCs, no ranging error can be observed. As a rough

\footnotetext{
${ }^{1}$ Note that, apart from a correlation receiver used for illustration in this paper, a large variety of other receiver architectures exist, e.g., half amplitude detectors. Nevertheless, the effects of the MPC on the ranging errors are very similar for all receiver architectures in use.
} 


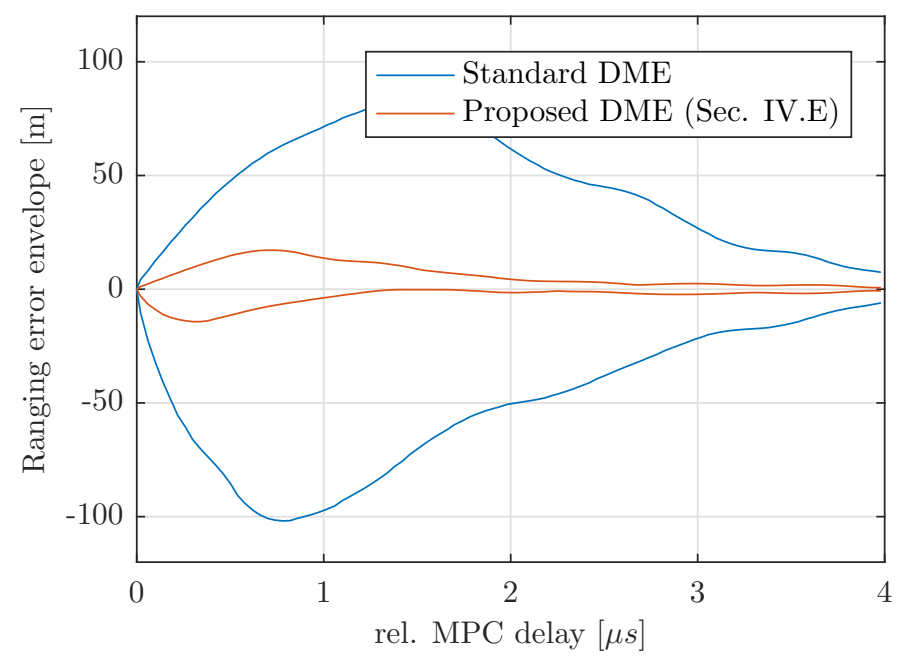

Fig. 4. Measured DME multipath ranging error envelope for a two-path channel model for a standard DME signal [22] and fast rise time $\cos -\cos ^{2}$ pulse (see Sec. IV). The relative MPC power is $-6 \mathrm{~dB}$.

approximation, ranging errors are usually not observed if the relative $\tau_{\mathrm{M} \text {,rel }}$ is smaller than $10 \%$ of the inverse of the signal bandwidth, e.g., $100 \mathrm{~ns}$ for the $1 \mathrm{MHz}$ system DME. Thus, for the typical navigation systems currently in use in civil aviation usually only lateral MPCs are able to cause ranging errors.

The ranging error scales $P_{\mathrm{M}, \mathrm{rel}}$ (not shown in Fig. 4): the larger the relative receive power of the MPC the larger the ranging error. Therefore, large ranging errors are to be expected when the receive power of the MPC is strong or when the LoS signal is attenuated. Whereas it is extremely rare that the receive power of a lateral MPC is as strong as the power of the undisturbed LoS signal, Sec. II discusses the effects which attenuate the LoS signal: ground MPCs, ground shadowing, and the aircraft pattern (also called the in situ antenna gain pattern). Depending on $P_{\mathrm{M} \text {,rel }}$ two situations can be distinguished:

- The MPC power is smaller than the LoS signal power, i.e., $P_{\mathrm{M}, \mathrm{rel}}<1$ : the ranging error due to one or more MPCs can be derived from a multipath ranging error envelope like the one shown in Fig. 4.

- The MPC has a higher power than the LoS, i.e., $P_{\mathrm{M}, \mathrm{rel}}>1$ : no upper bound on the ranging error exists. The receiver assumes the LoS signal to be the strongest signal component and, thus, falsely assumes the stronger MPC to be the LoS signal.

The Doppler frequency, i.e., the rate of change of the MPC's relative phase defines how fast the instant ranging error oscillates between the extrema of the ranging error envelope shown in Fig. 4. Depending on the employed receiver architecture, e.g., delay-locked loop (DLL), the estimated ranges may be averaged.

Nevertheless, the performance of any such algorithm employing averaging or carrier phase smoothing depends on the relative Doppler frequency. The inverse of the relative Doppler frequency $\nu_{\mathrm{M} \text {,rel }}$ directly determines for how long the range estimates have to be averaged in order to mitigate the ranging error.

\section{B. Critical Situations}

In the following, situations in which large ranging errors can be observed are described.

It is evident that a complete signal loss is extremely serious. A signal loss happens when the receive 


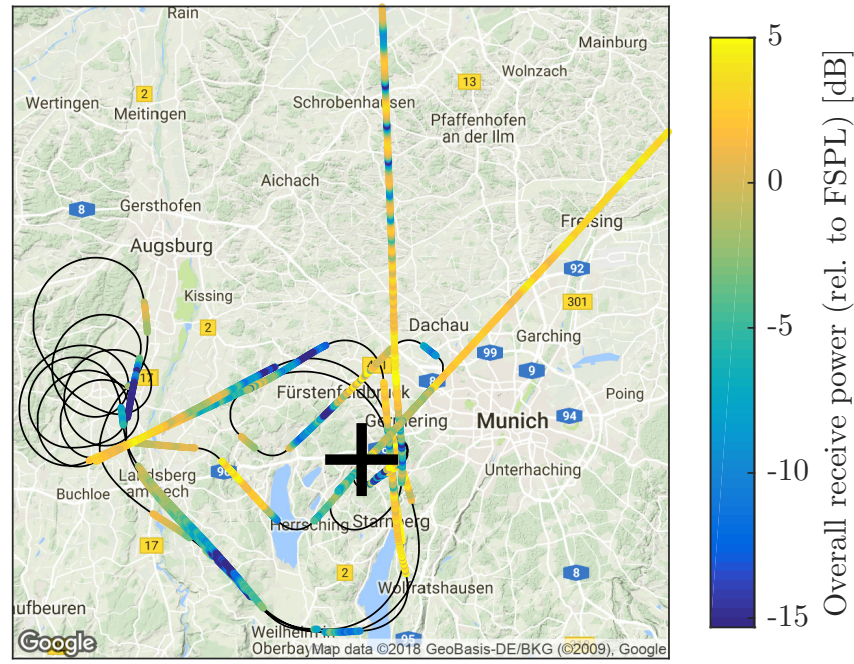

Fig. 5. Measured variation of the overall receive power (relative to free space path loss (FSPL)) due to multipath propagation (area size: $100 \mathrm{~km} \times 100 \mathrm{~km}$ ) [15]. The ground station position is marked by a black cross.

signal disappears or its receive power drops to such a low level that the receiver cannot acquire the signal any more. While such an event was not recorded during the conducted measurements from flying at distances closer than $100 \mathrm{~km}$, such a situation may still occur and should be considered. For example, situation of concern is that an aircraft flies at low level through a valley while there is a large mountain chain between aircraft and ground station. Another situation is that the aircraft is at far distance (larger than $100 \mathrm{~km}$ ) from the ground station and carries out a strong banking maneuver, thus attenuating the already weak signal components such that the receiver experiences a signal loss.

Apart from signal loss situations almost all other critical situations - which make up the vast majority of all critical cases - have in common that an MPC with large relative receive power exists. Each situation is analyzed using channel sounding measurement data. The high bandwidth of the channel sounding signal allows us to identify the different propagation paths.

Note that situations of low receive power where thermal noise is the main reason of inaccurate ranging estimates are not considered.

\section{B.1 Situation 1: Lateral MPCs and a Strong Ground MPC}

Due to the typically small relative delay $\tau_{\mathrm{M}, \text { rel }}$ of a ground MPC, the ground MPC usually interferes either constructively or destructively with the LoS signal resulting in the amplitude of the composite signal oscillating [16].

Fig. 5 shows the measured receive power as a function of the aircraft position. Strong fades of the receive LoS power exceeding $20 \mathrm{~dB}$ and lasting over $30 \mathrm{~s}$ can be attributed to ground multipath propagation [15]. Due to the resulting relative increase of lateral MPCs power, DME ranging errors exceeding $150 \mathrm{~m}$ have been attributed to ground multipath propagation in [23], [24]. From Fig. 5 it can be observed that flight trajectories tangential to the line aircraft-ground station can be especially critical as the receive signal may experience a long lasting fade. A reliable method to avoid long fades is to avoid these types of trajectories. 


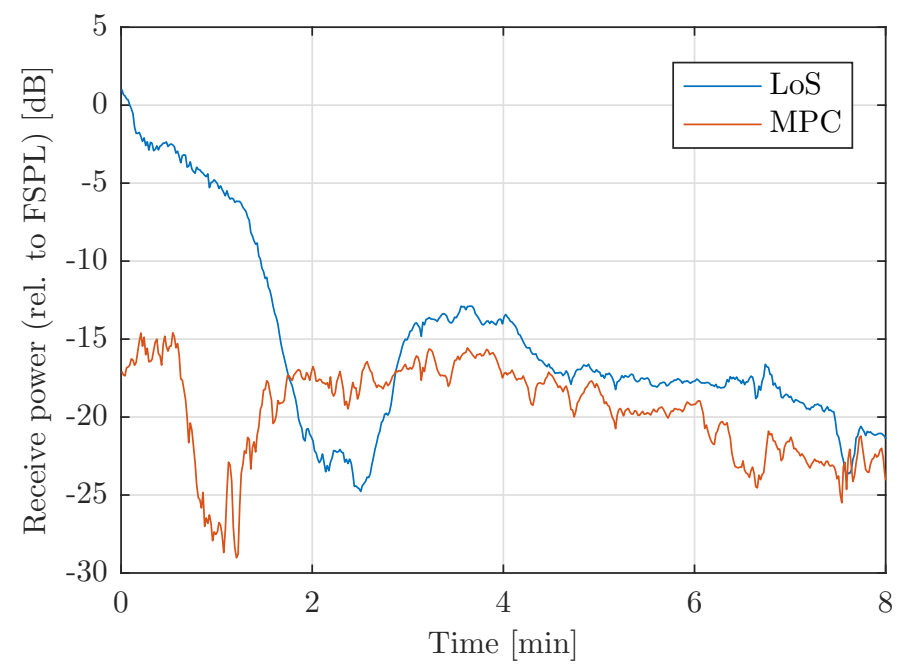

Fig. 6. Segment showing the effect of ground shadowing. The LoS signal is severely attenuated by vegetation, while the MPC maintains its original power level.

\section{B.2 Situation 2: Lateral MPCs and Shadowing of the LoS Signal}

Ground shadowing causes a strong attenuation or obliteration of the LoS signal. A good example of the possible consequences on ranging can be found in [8]. Herein, DME ranging errors exceeding $150 \mathrm{~m}$ are observed.

A later investigation using channel sounding experiments using the identical flight trajectory shows that the ranging error is caused by a very weak LoS signal in combination with a lateral MPC as visualized in Fig. 6 [10]. While the LoS signal experiences a significant attenuation, the lateral MPC maintains its power level over most of the time. Applying a method to localize reflectors [19] shows that the lateral MPC is caused by a reflection off a building in the vicinity of the ground station as shown in Fig. 7. Due to the location of the building, the lateral MPC passes freely beside the same trees blocking the LoS signal. The result is a lateral MPC, visible for more than 8 minutes and exceeding the LoS signal in terms of power by more than $5 \mathrm{~dB}$ for part of the time. The most reliable ways to avoid shadowing is to avoid flying at low elevation angles (as seen from the ground station) or to place the ground antenna at a larger height.

\section{B.3 Situation 3: Lateral MPCs and Aircraft Pattern}

In the following a situation is analyzed in which ranging errors exceeding $200 \mathrm{~m}$ can be attributed to the influence of the aircraft pattern [24]. In the analyzed flight segment the aircraft is flying at a distance of 2 to $4 \mathrm{~km}$ from the ground station and is performing a long and steep turn. The signal is acquired using a standard DME dipole L-Band antenna mounted on a Falcon 20E.

Fig. 8 shows the receive power of both the LoS signal and the strongest lateral MPC. Before the aircraft starts banking $(t<5 \mathrm{~s})$, the relative power of the strongest lateral MPC is roughly $-18 \mathrm{~dB}$ - a situation which results in a very minor ranging error. As the aircraft starts banking the LoS signal is severely attenuated due to the aircraft pattern. Most of the time the power of the strongest lateral MPC is attenuated in a similar fashion as the LoS signal, an indication that both signals are received from a similar direction. Thus, for most of the time the relative power of the strongest lateral MPC experiences only small changes: the consequence is that the ranging error stays small. However, the situation becomes critical around $t=45 \mathrm{~s}$. The strongest lateral MPC is received from a different direction as the LoS signal. Thus, the lateral MPC is not attenuated by the aircraft 


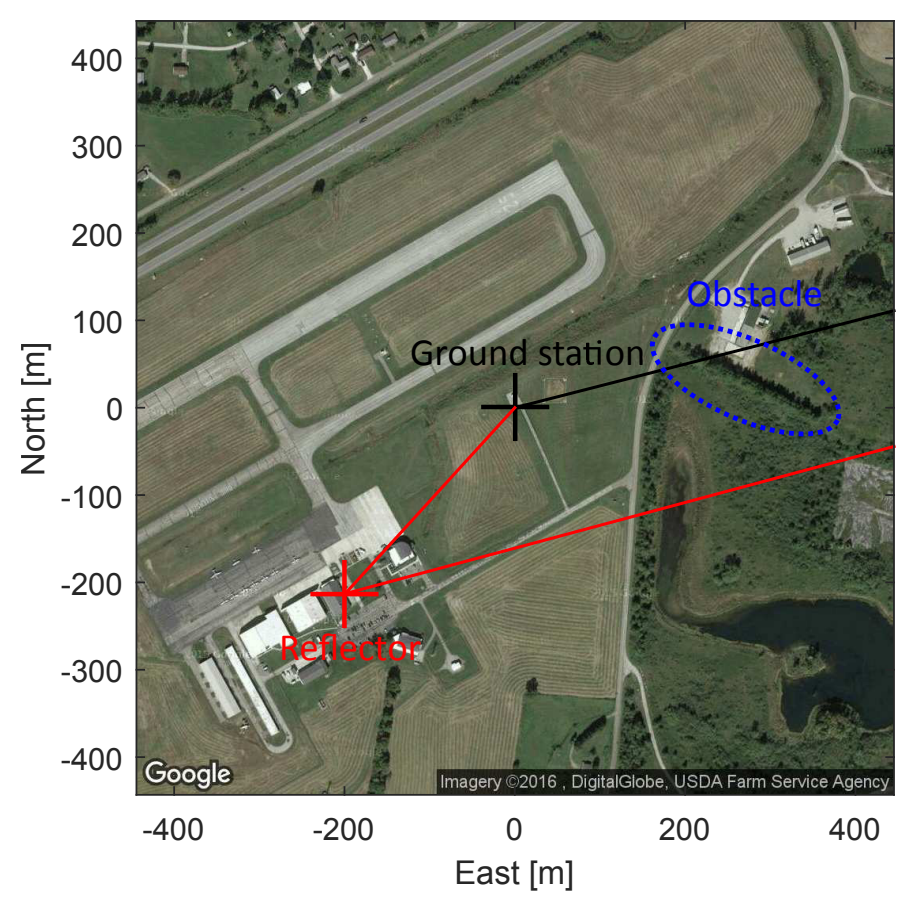

Fig. 7. Locations of the reflector and the ground station. The aircraft is flying in the east of the ground station as indicated by the two lines connecting reflector and ground station with the aircraft.

pattern and, hence, a large ranging error arises.

\section{Summary of Effects}

Tab. I summarizes the critical propagation effects and their resulting effects on the receive signal.

\section{Mitigation techniques}

Mitigating multipath to the level desired for future aviation requires a range of mitigation techniques to accommodate different users and ground station technologies. Terrestrial navigation will mostly be based on existing signals such as DME or signals designed for other purposes such as ADS-B or LDACS. So both techniques suitable for existing users to support near term benefits and more powerful ones for future use are needed. Techniques that will require upgraded ground infrastructure will take significant time to become available and operational. In other words, different mitigation techniques are needed for different classes of users and installed equipment. At the same time, most of the techniques can be employed in combination with each other creating more powerful mitigation.

This section covers several classes of mitigation using DME as the example terrestrial navigation signal. They represent different challenges and possibilities in terms of the changes needed in the user, avionics and ground equipment. These are presented roughly in order of least to most equipment changes required to obtain meaningful benefits. 


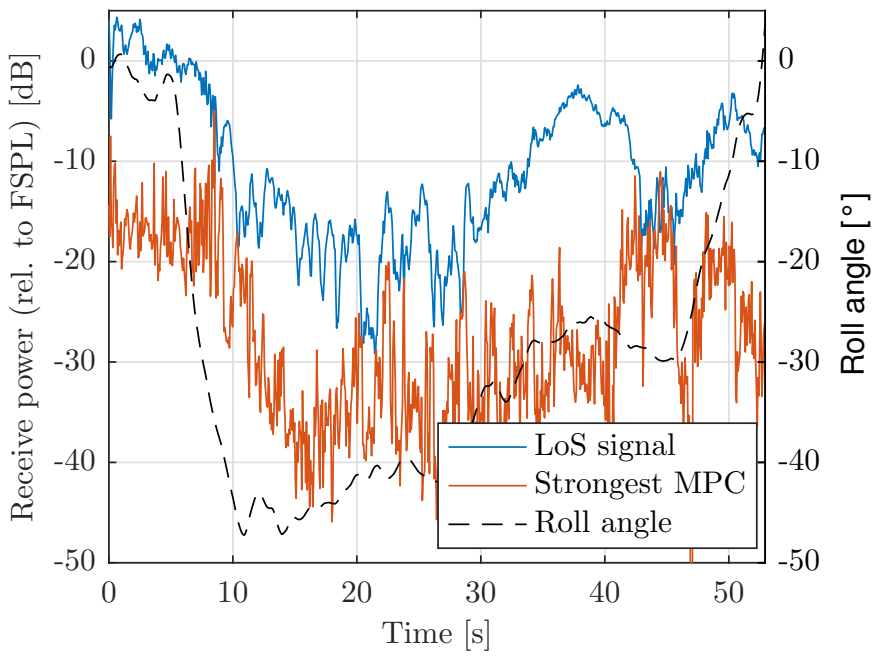

Fig. 8. Attenuation of receive power due to strong banking of the aircraft.

TABLE I. Summary of relevant propagation effects.

\begin{tabular}{|l|l|l|l|l|}
\hline Effect & Physical Cause & $\begin{array}{l}\text { Resulting Effect on } \\
\text { Receive Signal }\end{array}$ & $\begin{array}{l}\text { Scenarios of Major } \\
\text { Concern }\end{array}$ & $\begin{array}{l}\text { Scenarios of Minor } \\
\text { Concern }\end{array}$ \\
\hline \hline $\begin{array}{l}\text { Ground } \\
\text { MPC }\end{array}$ & Reflection off ground & $\begin{array}{l}\text { Severe variation of } \\
\text { overall receive power } \\
\text { with long lasting } \\
\text { fades }\end{array}$ & $\begin{array}{l}\text { Flight trajectories } \\
\text { tangential to the line } \\
\text { aircraft-ground } \\
\text { station }\end{array}$ & $\begin{array}{l}\text { Flying directly } \\
\text { towards/away from } \\
\text { ground station }\end{array}$ \\
\hline $\begin{array}{l}\text { Lateral } \\
\text { MPCs }\end{array}$ & $\begin{array}{l}\text { Reflections off } \\
\text { buildings, trees and } \\
\text { other objects }\end{array}$ & $\begin{array}{l}\text { MPCs able to cause } \\
\text { ranging errors }\end{array}$ & $\begin{array}{l}\text { Low height of ground } \\
\text { station }\end{array}$ & $\begin{array}{l}\text { Highly elevated } \\
\text { antenna position }\end{array}$ \\
\hline $\begin{array}{l}\text { LoS } \\
\text { signal } \\
\text { and } \\
\text { ground } \\
\text { shadow- } \\
\text { ing }\end{array}$ & $\begin{array}{l}\text { Shadowing by } \\
\text { buildings or terrain }\end{array}$ & $\begin{array}{l}\text { Attenuation of LoS } \\
\text { signal }\end{array}$ & $\begin{array}{l}\text { Aircraft at low } \\
\text { elevation angles (seen } \\
\text { from ground station) }\end{array}$ & $\begin{array}{l}\text { Aircraft at higher } \\
\text { elevation angles (seen } \\
\text { from ground station) }\end{array}$ \\
\hline $\begin{array}{l}\text { Aircraft } \\
\text { pattern }\end{array}$ & $\begin{array}{l}\text { Antenna radiation } \\
\text { patterns, airframe }\end{array}$ & $\begin{array}{l}\text { Attenuation of LoS } \\
\text { signal and MPCs }\end{array}$ & $\begin{array}{l}\text { Banking aircraft } \\
\text { close to ground } \\
\text { station }\end{array}$ & Non-banking aircraft \\
\hline
\end{tabular}

\section{A. Operational Changes}

An easy mitigation, in terms of equipment changes, is to change user behavior. One could develop flight operations and procedures to account for areas of significant multipath propagation. Thus no equipment changes are needed. The challenge with this mitigation is to determine areas of severe multipath propagation and develop acceptable changes in procedures. Operational changes are credible, as multipath from terrestrial signal sources is a reasonably static phenomenon. As previously discussed, the multipath of concern is caused by reflections from static objects or the ground. Flight survey is one means to identify potential areas of issue. Regular flight inspection by the air navigation service provider (ANSP) is used to inspect current ground navigation aids. However, regular flight inspection is both time consuming and expensive (to cover a large area) and results are subject to change with environment changes. Another means is to crowd-source information. In the case of DME, there are many aircraft equipped with both GNSS and DME. 


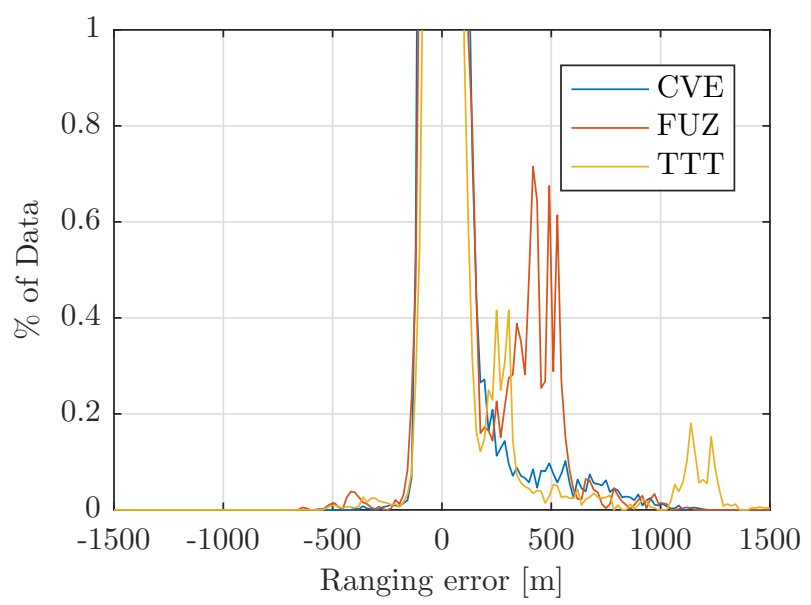

(a) Raw

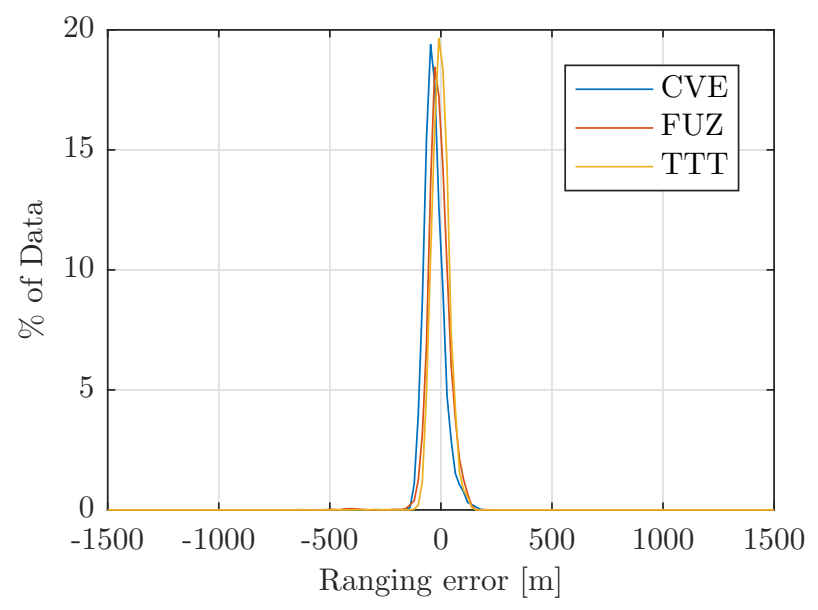

(b) Screened

Fig. 9. DME raw and screened ranging error distribution for Dallas Fort Worth DMEs (CVE, FUZ, TTT) [26].

One could examine discrepancies in DME and GNSS estimated ranges to identify potential regions of concern. A third method is to use modeling to identify potential areas of multipath for inspection. A model for identifying critical areas causing MPCs with critical delays and relative Doppler velocity that are both significant and difficult to mitigate was developed [25]. For these latter two methods, flight inspection can then be used judiciously to verify and precisely determine the region and level of multipath.

Once areas of high multipath are identified, the mitigation would be to alter the use of the signals in these areas. Several changes are possible: Inflate integrity bounds to account for increase in error, provide a location-dependent multipath correction, restrict use of the signal at those locations, or modify the surrounding terrain to reduce the multipath effects.

Fig. 9(a) shows the distribution of ranging errors observed during FAA flights near Dallas/ Fort Worth International Airport (DFW) in April and May of 2011. DFW has three local navaids offering distance-measurement service, Maverick VOR/DME (TTT), Ranger VORTAC (FUZ) and Cowboy VOR/DME (CVE). In addition to the roughly Gaussian central distribution between -150 and $150 \mathrm{~m}$, there are outliers that exceed $200 \mathrm{~m}$ for each station. These outliers are generally due to errors where the measured range exceeds the actual. This suggested that they may be the result of multipath-induced delays. In examining the data, it is noticed that the errors correspond to lowaltitude portions of the flight path, and even measurements collected during ground operations..

A potential operational mitigation is altitude limits below which the signal is not to be used. Fig. 9(b) shows that same data except it has been screened to eliminate measurements made below $400 \mathrm{ft}$ above ground level (AGL) and weaker than $-90 \mathrm{dBm}$.

\section{B. Receiver Detection \& Exclusion}

Measurements made by the receiver can be useful in identifying severe multipath. Several general techniques involving comparison checking can be employed. Comparisons with redundant, prior or model-derived measurements can be employed.

A classic error detection method is to perform redundancy checks. Aviation redundancy algorithms such as receiver autonomous integrity monitoring (RAIM) and advanced RAIM (ARAIM) may be used. Analysis of the utility of their detection level relative to nominal error is needed. As discussed previously, there are scenarios where multipath from a given station is more or less likely. 
Hence, some measurements can be weighed more heavily than others or even identified as trusted. This would aid with exclusion and avoid false alarms.

Even without redundant measurements there are several features that can be used for comparison and multipath mitigation. One method would to compare received signal strength to the anticipated given FSPL. Fading and interference by multipath can alter the signal strength. However, other factors such as banking, as discussed previously, can also cause a degradation. As more powerful software receivers are available, a more intensive metric is to examine the signal itself and compare it to model waveforms. Significant deviations may indicate multipath situations. Supervised learning on a model set of LoS plus MPC that span anticipated multipath cases to identify and mitigate multipath errors may provide utility [27]. Another metric is to compare with past measurements. For example, examine the continuity of the range solution for significant jumps. Aircraft have very specific dynamic behavior. That behavior can be used to detect unusual jumps in the range that would indicate a MPC induced error. An aircraft in the terminal area, where accuracy requirements are higher, flies significantly slower than an en route aircraft. As an aircraft at $200 \mathrm{kt}$ (knots) travels about $100 \mathrm{~m} / \mathrm{s}$, range jumps on the order of $100 \mathrm{~m}$ should be noticeable with $1 \mathrm{~Hz}$ or better measurements. The challenges with these techniques are false alarms and missed detection.

\section{Receiver Averaging}

For DME, averaging is applied on the round trip time, mitigating the multipath on both the interrogation and reply (or down and up) transmission channels. Averaging is a traditional means of mitigating multipath and other fluctuating errors. Given motion of the aircraft, averaging can be used to cancel out much of the multipath induced error. Averaging works on multipath because multipath effects vary with the multipath delay. The variation of the ranging error depends of the relative Doppler frequency of the MPC $\nu_{\mathrm{M} \text {,rel }}$ causing the error. For example, if the relative of an $\mathrm{MPC}$ is $\nu_{\mathrm{M} \text {,rel }}=1 \mathrm{~Hz}$, the resulting range estimate will oscillate around the true value with a frequency of $1 \mathrm{~Hz}$. Thus, the relative Doppler frequency essentially quantifies the variation of multipath delay as described in Sec. II.

In the case of DME, current flight management systems (FMS) conduct some filtering of its ranges to update the inertial system. Fig. 10 shows an example scenario to illustrate the effect of averaging. The multipath error curve from Fig. 4 is used as the underlying model for multipath error. In this scenario, the aircraft flies in a direction where it is constantly exposed to multipath with a constant $P_{\mathrm{M} \text {,rel }}=6 \mathrm{~dB}$ direct-to-multipath signal power level assumed. The aircraft flies in this direction at $100 \mathrm{~m} / \mathrm{s}$ or $194 \mathrm{kt}$. Given the initial position shown in the figure, the relative MPC delay $\tau_{\mathrm{M} \text {,rel }}$ is initially $465 \mathrm{~m}$ (or $1.55 \mu \mathrm{s}$ ) with a rate of change approximately $35 \mathrm{~m} / \mathrm{s}$ (or $\nu_{\mathrm{M}, \mathrm{rel}}=120 \mathrm{~Hz}$ ). Fig. 11(a) shows the multipath error for the next $20 \mathrm{~s}$ of flight with the blue, red, and yellow curves indicating instantaneous, $T_{\mathrm{av}}=2 \mathrm{~s}$ averaged, and $T_{\mathrm{av}}=10 \mathrm{~s}$ averaged multipath error, respectively. Given this motion and averaging, the multipath induced error is reduced from about $70 \mathrm{~m}$ (instantaneous) to about $10 \mathrm{~m}\left(T_{\mathrm{av}}=2 \mathrm{~s}\right)$ or less $\left(T_{\mathrm{av}}=10 \mathrm{~s}\right)$. $10 \mathrm{~s}$ averaging would require clock stability of roughly $10 \mathrm{~ns} / \mathrm{s}$. The avionics would require a better oscillator than a crystal oscillator (XO). A good and recently calibrated temperature compensated XO (TCXO) is likely the minimum required.

The averaging is effective because over this period, the aircraft goes through numerous peak to trough cycles of multipath. This does not happen for all cases and depends on geometry. Fig. 10 shows also scenario 2 where the geometry results in a slow rate of change in multipath delay. This is the case with a shallow multipath reflection. This scenario uses an aircraft traveling at same $100 \mathrm{~m} / \mathrm{s}$ speed. In this scenario, the aircraft at its initial position experiences multipath with a delay of $79 \mathrm{~m}$ 


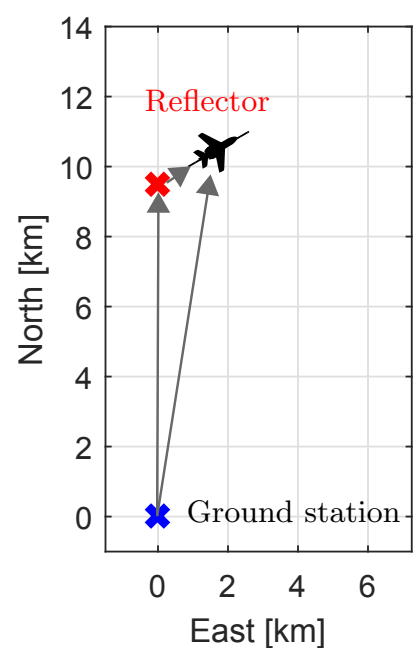

a) Scenario 1

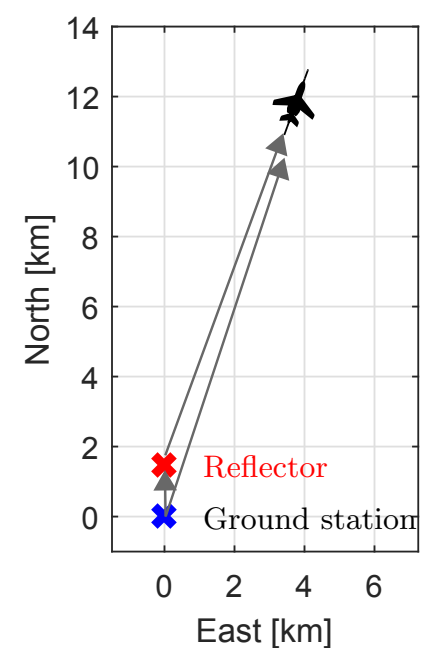

b) Scenario 2

Fig. 10. Signal \& multipath geometry for the two scenarios (aircraft movement over $20 \mathrm{~s}$ shown).

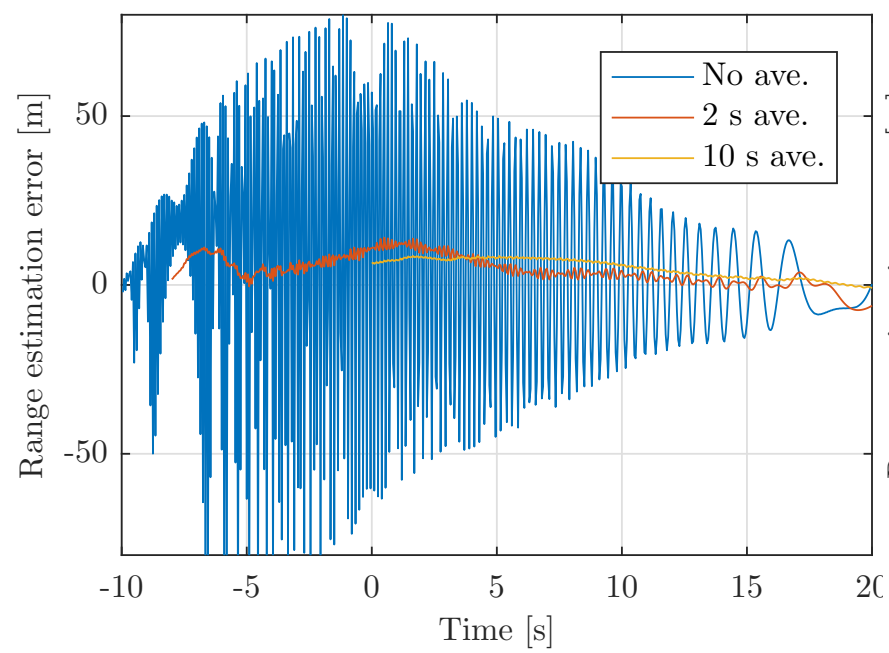

(a) Scenario 1

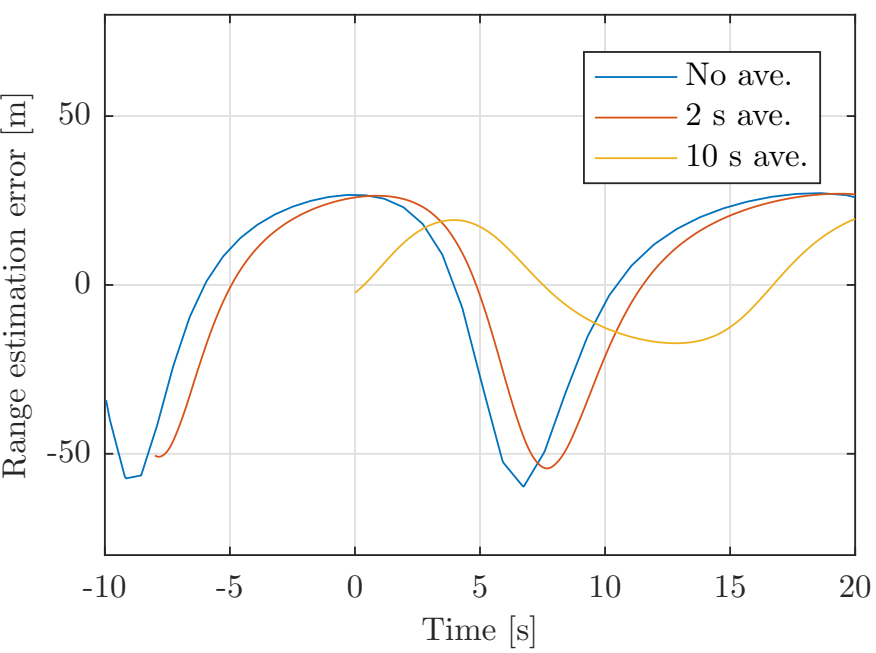

(b) Scenario 2

Fig. 11. Multipath induced error over 20 seconds for both scenarios with averaging (none, $T_{\mathrm{av}}=2 \mathrm{~s} \& T_{\mathrm{av}}=10 \mathrm{~s}$ ).

(or $0.263 \mu \mathrm{s}$ ) and a delay rate of change of less than $0.1 \mathrm{~m} / \mathrm{s}\left(\right.$ or $\nu_{\mathrm{M}, \mathrm{rel}}=0.3 \mathrm{~Hz}$ ). Fig. 11(b) shows the resulting multipath error experienced.

In this case, $T_{\mathrm{av}}=2 \mathrm{~s}$ averaging does very little, with maximum error still over $50 \mathrm{~m} . T_{\mathrm{av}}=10 \mathrm{~s}$ averaging does decrease the maximum error to slightly over $20 \mathrm{~m}$. Furthermore, one can imagine geometries that are worse where the multipath varies even more slowly.

A natural solution if $T_{\mathrm{av}}=10 \mathrm{~s}$ averaging is not enough is to further extend the averaging time. Extended averaging is possible but it requires a much more stable source of time than typically carried in todays avionics. One means for mitigation is to use a better oscillator. Another is for the ground system to provide a stable carrier signal which the user can track. This essentially transfers the stability of the ground clock to the aircraft. Like simple averaging, it is effective on multipath on both the interrogation and reply signals. For DME, this technique would require either a more stable oscillator on the ground or in the avionics [22]. 


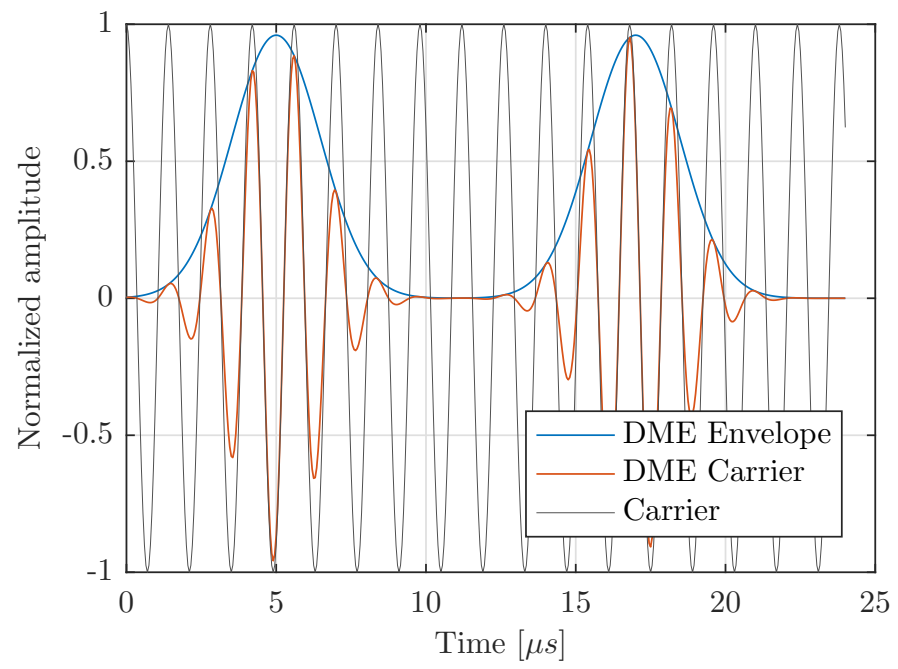

Fig. 12. DME pulse pair \& underlying carrier.

\section{Carrier-Smoothed Code}

Carrier phase typically has less susceptibility to multipath than the envelope or code of the transmitted signal. carrier smoothed code (CSC), a technique commonly used in GNSS, can also be employed for DME provided a consistent carrier measurement can be made. Equation (1) shows the Hatch filter implementation where $R$ is the code based range (or pseudo range), $\Phi$ is the carrier measurement, $\hat{R}$ is the CSC range, $n$ is the discrete smoothing window length (in terms of samples), and $k$ is the discrete sequential sample number.

$$
\hat{R}(k)=\frac{1}{n} R(k)+\frac{n-1}{n}[(k-1)+(\Phi(k)-\Phi(k-1))]
$$

Carrier-smoothed code is feasible on DME provided that the underlying DME signal is generated by a continuous carrier and if that carrier is stable enough. Fig. 12 illustrates the basic assumption. With about 3000 pulse pairs per second (PPPS), there is a signal on average every $330 \mu$ s for tracking.

DME ground systems generally do use a continuous carrier between pulses. However, todays DMEs and TACANs generally do not have adequate clock stability for carrier phase processing to improve multipath performance. Examination of the stability of the Woodside DME (VORTAC) supports this generalization [22]. Similar results were found in [28]. Hence, using CSC on DME requires that new and existing ground stations utilize more stable oscillators. Another limit of this technique for DME is that it is only useful on ground-to-air (reply) signals and does not mitigate multipath on air-to-ground (interrogation) signals. The ground receives transmissions from various aircraft which all look similar and do not have a stable or common carrier. Hence, the ground station cannot track and smooth the carrier from each individual aircraft using the station.

\section{E. Increase Signal Bandwidth}

Another mitigation is to use a sharper signal that is, increase signal bandwidth. This can entail several requirements. First, the new signal needs to be interoperable with the signal in space definition and requirements. Second, it needs to be interoperable with the existing signal; that is, legacy avionics processing the new signal should not suffer any adverse effects. 


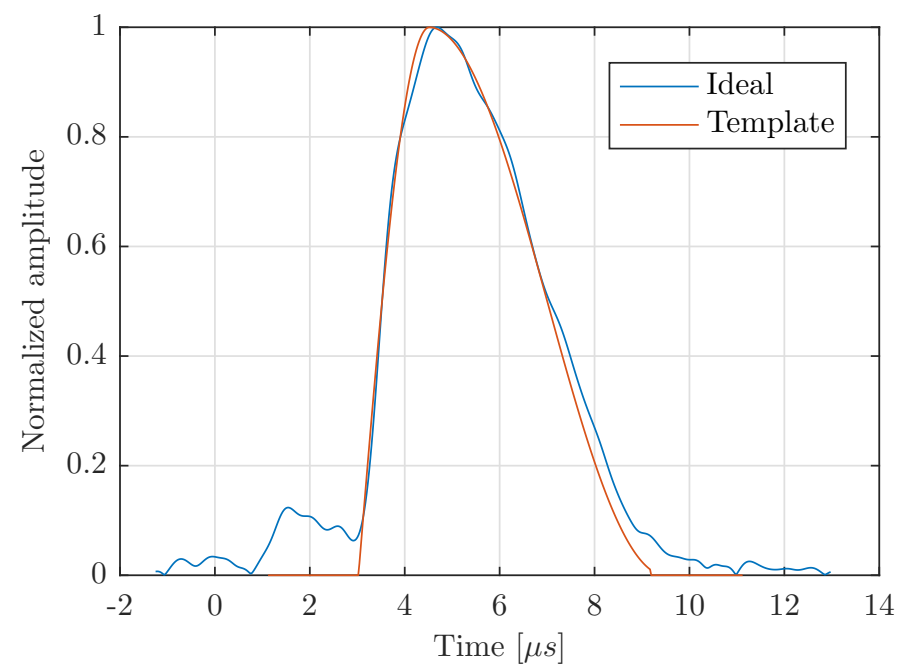

Fig. 13. Fast rise time $\left(\cos -\cos ^{2}\right)$ DME pulse.

For DME signals, there is some flexibility in its pulse design requirements. Several fast rise-time pulses were implemented and tested on a modified DME/TACAN ground system at the FAA Technical Center to quantify the benefits. The pulse shapes implemented complied with international standards [6]. Some were consistent with DME/N standards while others did not comply with DME/N standards but were acceptable under DME/P standards.

Fig. 13 shows the measured on-air signal for fastest rise time pulse tested. It is compatible with DME/P standards. Fig. 4 shows multipath error for $P_{\mathrm{M}, \mathrm{rel}}=6 \mathrm{~dB}$ direct-to-multipath signal power ratio when using this pulse. Relative to the normal DME pulse whose multipath error curve is seen in Fig. 4, the fast rise-time pulse significantly reduces the overall error with maximum error reduced by over half. The maximum error is about $50 \mathrm{~m}$. The fast rise time pulse can be used with other mitigation techniques such as averaging.

Another proposed DME pulse design is the Smoothed Concave Polygon (SCP). Versions of this design have been developed that meet ICAO DME requirements while also reducing multipath induced errors. Analysis has shown it to reduce ranging errors by $36-77 \%$ [27], [29].

\section{WAY FORWARD}

Sec. II gave an overview of how the AG channel characteristics can impact navigation performance. Sec. IV describes how the impact of the channel on the range estimation may be minimized. The following section defines different scenarios causing large ranging errors and describes how well the resulting ranging errors may be mitigated using methods from Sec. IV.

Fig. 14 shows different scenarios and provides methods for mitigating ranging errors. The scenarios are ordered by their anticipated probability of occurrence, from high to low.

Scenario 1 is very typical: a lateral MPC with $P_{\mathrm{M} \text {,rel }}<1$, which is usually the case for lateral MPCs, is degrading the range estimation. Given a suitable geometry, which should usually be the case [25], its influence on the range estimation can be averaged out and the error significantly reduced without a requirement for upgraded avionics clocks..

Scenario 2 represents the situation when the LoS path is strongly attenuated. The drop in received LoS power may be due to shadowing by a building or terrain, ground multipath, or a banking aircraft. Due to the attenuation of the LoS path, a lateral MPC may exceed the LoS path in terms of power, i.e. $P_{\mathrm{M} \text {,rel }} \geq 1$. The best way to minimize the impact of the resulting ranging error 


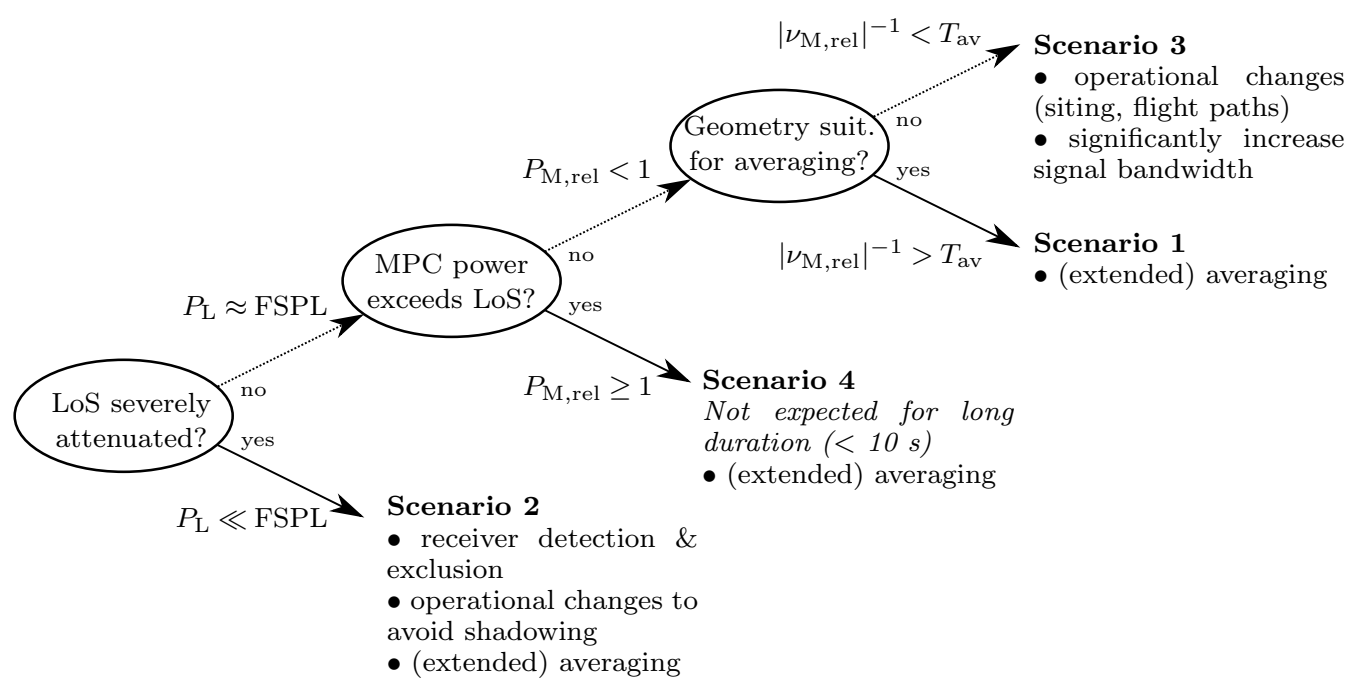

Fig. 14. Mitigation of ranging errors for different propagation scenarios.

on the overall navigation performance is to detect the low power level of the LoS path. The receiver can then exclude the corresponding ground station. Operational changes can allow mitigation of the error, e.g., by making sure that no shadowing appears. Additionally averaging may help, if the situation only persists for a short duration relative to the averaging time $T_{\mathrm{av}}$. Nevertheless, the longer the situation persists, averaging becomes less effective.

Scenario 3 is very similar to scenario 1 . However, it is expected to appear with a significantly lower probability than scenario 1, as it requires a geometry unsuitable for averaging [25]. The most economic way is to avoid the situation by operational changes. Those changes could be a slight change of the flight path or removing reflecting objects from the surroundings of the ground station, as outlined in [25].

Scenario 4 is only expected to appear with a very low probability, as it requires a very strong lateral MPC. Lateral MPCs with $P_{\mathrm{M}} \approx$ FSPL are a very unlikely event as indicated in Fig. 2. As such lateral MPCs are expected to persist only for a short time, e.g., a few seconds, their influence on the range estimation can be averaged out.

\section{Conclusions}

In this paper a comprehensive analysis of the air-ground (AG) channel and its effects on the ranging performance of terrestrial radionavigation are presented. The paper shows that propagation characteristics of the AG channel can significantly degrade the range estimation. The degradation usually occurs due to an unfavorable reception of the LoS signal and MPCs: most severe are situations where the LoS signal is attenuated and very strong MPCs are present. The resulting ranging errors from such occurrences have been observed to exceed $200 \mathrm{~m}$.

The paper explores different classes of mitigation, each with varying capabilities and requiring different changes in hardware or software. Some mitigation techniques can be achieved without any equipment changes while others require new avionics and ground equipment. The benefit of having an organized classification allows us to choose the best set of mitigations given the requirements and the limitations of the system. 


\section{ACKNOWLEDGMENTS}

First and foremost, the authors want to express their gratitude towards Wouter Pelgrum, Elisabeth Nossek, and Okuary Osechas for the realization of the 2015 flight trials in Athens, OH and the provision of the measurement data. Additionally, we would like to thank the U.S. Federal Aviation Administration (FAA) and Mr. Robert Erikson for the provision of the Dallas-Fort Worth measurement data and results, which illustrated important APNT factors related to DME performance and flight-inspection measurement. The authors want to express thanks to the entire Institute of Communication and Navigation as well as the Institute of Flight Operations at DLR for their support during the flight measurements.

\section{REFERENCES}

[1] L. Eldredge, P. Enge, M. Harrison, R. Kenagy, S. Lo, R. Loh, R. Lilley, M. Narins, and R. Niles, "Alternative positioning, navigation and timing (PNT) study," in International Civil Aviation Organisation Navigation Systems Panel (NSP), Montreal, Canada, 2010.

[2] W. E. Jackson, The Federal Airways System. Washington DC: The Institute of Electrical and Electronic Engineers, 1970.

[3] M. Schnell, U. Epple, D. Shutin, and N. Schneckenburger, "LDACS: future aeronautical communications for air-traffic management," IEEE Commun. Mag., vol. 52, no. 5, pp. 104-110, 2014.

[4] Y.-H. Chen, S. Lo, S.-S. Jan, G.-J. Liou, D. Akos, and P. Enge, "Design and Test of Algorithms and Real-Time Receiver to use Universal Access Transceiver ( UAT) for Alternative Positioning Navigation and Timing (APNT )," in Proc. ION GNSS+, Tampa, FL, 2014.

[5] S. Lo, Y.-H. Chen, S. Zhang, and P. Enge, "Hybrid APNT: terrestrial radionavigation to support future aviation needs," in Proc. ION GNSS+, Tampa, FL, 2014.

[6] International Civil Aviation Organization (ICAO), "International Standards and Recommended Practices, Annex 10 to the Convention on International Civil Aviation, Volume I Radio Navigation Aids," Tech. Rep., 2006.

[7] S. Lo, Y.-H. Chen, P. Enge, B. Peterson, and R. Erikson, "Distance Measuring Equipment Accuracy Performance Today and for Future Alternative Position Navigation and Timing (APNT)," in Proc. ION GNSS+, Nashville, TN, 2013.

[8] W. Pelgrum and K. Li, "An investigation on the contributing factors of enhanced DME ranging errors," in Proc. ION GNSS+, Tampa, FL, 2015.

[9] N. Schneckenburger, T. Jost, D. Shutin, M. Walter, T. Thiasiriphet, M. Schnell, and U.-C. Fiebig, "Measurement of the L-band air-to-ground channel for positioning applications," IEEE Trans. Aerosp. Electron. Syst., vol. 52, no. 5, pp. 2281-2297, 2016.

[10] O. Osechas, N. Schneckenburger, W. Pelgrum, and E. Nossek, "Characterization of the Groundto-Air Ranging Performance of the 960-1215 MHz ARNS Band Using OFDM Measurements in the 902-928 MHz ISM Band," in Proc. ION ITM, Monterey, CA, 2016.

[11] T. J. Willink, C. C. Squires, G. W. Colman, and M. Muccio, "Measurement and characterization of low-altitude air-to-ground MIMO channels," IEEE Trans. Veh. Technol., vol. 65, no. 4, pp. 2637-2648, 2016.

[12] D. W. Matolak and R. Sun, "Air-ground channel characterization for unmanned aircraft systems - part I: methods, measurements, and models for over-water settings," IEEE Trans. Veh. Technol., vol. 66, no. 1, pp. 26-44, 2016. 
[13] R. Sun and D. W. Matolak, "Air-ground channel characterization for unmanned aircraft systems - part II: hilly \& mountainous settings," IEEE Trans. Veh. Technol., vol. 66, no. 3, pp. 1913-1925, 2017.

[14] D. W. Matolak and R. Sun, "Air-ground channel characterization for unmanned aircraft systems - part III: the suburban and near-urban environments," IEEE Trans. Veh. Technol., vol. PP, no. 8, pp. 6607-6618, 2017.

[15] N. Schneckenburger, T. Jost, D. Shutin, and U.-C. Fiebig, "Line of sight power variation in the air to ground channel," in Proc. EuCAP, Davos, Switzerland, 2016.

[16] J. D. Parsons, The Mobile Radio Propagation Channel, 2nd. West Sussex, United Kingdom: Wiley, 2000.

[17] ITU-R, "P.1008-1: Reflection from the surface of the Earth," Tech. Rep., 1990.

[18] — "P.527-3: Electrical characteristics of the surface of the earth," Tech. Rep., 1992.

[19] N. Schneckenburger, T. Jost, D. Shutin, M. Walter, U.-C. Fiebig, and G. Del Galdo, "Reflector localization for geometrical modeling the air-ground channel," IEEE Trans. Veh. Technol. (submitted for publication), 2017.

[20] M. Schwinzerl, T. Jost, F. Pérez-Fontán, M. Schoenhuber, W. Wang, M. Walter, and T. Pelzmann, "Wide-Band Characterization of Antennae Plus Aircraft Platform Patterns in Land Ka-Band," in Proc. EuCAP, Lisbon, Portugal, 2015, pp. 1-5.

[21] K. J. Keeping and J. C. Sureau, "Scale model pattern measurements of aircraft l-band beacon antennas," Lincoln Laboratory, Tech. Rep., 1975.

[22] S. Lo, Y.-H. Chen, B. Segal, B. Peterson, P. Enge, R. Erikson, and R. Lilley, "Containing a difficult target: techniques for mitigating DME multipath to alternative position navigation and timing (APNT)," in Proc. ION ITM, San Diego, CA, 2014.

[23] K. Li, W. Pelgrum, and A. Naab-Levy, "Impact of ground multipath on terrestrial radio navigation performance," in Proc. ION GNSS+, Nashville, TN, 2013, pp. 722-736.

[24] T. Thiasiriphet, N. Schneckenburger, and M. Schnell, "Ranging with LDACS: results from measurement campaign," in Proc. ICNS, Herndon, VA, 2016.

[25] N. Schneckenburger, S. Lo, M. Walter, and U.-C. Fiebig, "Geometric rules for terrestrial radionavigation multipath mitigation by averaging," Navigation, vol. 64, no. 2, pp. 231-236, 2017.

[26] R. Erikson and R. Lilley, "Analysis of Dallas-Fort Worth area flight data for APNT DME positioning," FAA, Tech. Rep., 2014.

[27] E. Kim, "Enhancing DME/N Multipath Rejection with Tightened Pulse Waveform Variation Suppression," in Proc. IEEE/AIAA DASC, East Syracuse, NY, 2013.

[28] K. Li and W. Pelgrum, "Flight test evaluation of enhanced DME (eDME) performance enhancements," in Proc. ION GNSS+, Nashville, TN, 2012.

[29] E. Kim and J. Seo, "SFOL pulse: a high accuracy DME pulse for alternative aircraft position and navigation," Sensors, vol. 17, no. 10, p. 2183, 2017. 